International Journal of Clinical Dermatology \& Research (IJCDR)

ISSN 2332-2977

\title{
Phenytoin-Induced Hypersensitivity Syndrome Presenting on the Palms and Soles of an 18-Year-Old Woman
}

Case Report

Kallini JR ${ }^{1 *}$, Jochen $\mathrm{T}^{2}$, Ukpong $\mathrm{C}^{3}$

${ }^{1}$ Department of Internal Medicine, Eisenhower Medical Center, Rancho Mirage, California, and Saint Louis University, USA.

${ }^{2}$ Department of Dermatology, University of Southern California, Los Angeles, California; Department of Dermatology, Eisenhower Medical Center, Rancho Mirage, California; and Contour Dermatology \& Cosmetic Surgery Center, Rancho Mirage, California, USA

${ }^{3}$ Department of Internal Medicine, Eisenhower Medical Center, Rancho Mirage, California, USA

Key words: Hypersensitivity Syndrome; Phenytoin-Induced.

\section{*Corresponding Author:}

Kallini JR,

39000 Bob Hope Dr., Rancho Mirage, CA, USA.

Tel: (760) 3403911 Ext:5457; Fax: (760) 8378581

E-mail: jrkallini@gmail.com

Received: June 11, 2014

Accepted: July 07, 2014

Published: July 09, 2014

Citation: Kallini JR, Jochen T, Ukpong C (2014) Phenytoin-Induced Hypersensitivity Syndrome Presenting on the Palms and Soles of an 18-Year-Old Woman. Int J Clin Dermatol Res. 2(4), 21-24. doi: http:// dx.doi.org/10.19070/2332-2977-140006

Copyright: Kallini JR (C) 2014. This is an open-access article distributed under the terms of the Creative Commons Attribution License, which permits unrestricted use, distribution and reproduction in any medium, provided the original author and source are credited.

\section{Introduction}

Anticonvulsant drug-induced hypersensitivity syndrome (DIHS) is a disorder that occurs in some patients taking anticonvulsant medications. Fever is the most common feature, seen in $90-100 \%$ of cases. It is also characterized by rash, hepatitis, and other multiorgan involvement. The mechanism is unknown. Implicated anticonvulsants include those in the phenytoin category, carbamazepine, and lamotrigine[1]. This syndrome is now most commonly known as Drug Reaction (or Rash) with Eosinophilia and Systemic Symptoms (DRESS), a term more descriptive of its presentation. It is also known as Drug-Induced Delayed Multiorgan Hypersensitivity Syndrome (DIDMOHS) and Drug-Induced Pseudolymphoma. Anticonvulant hypersensitivity syndrome is an older term that has lost favor due to the fact that, aside from antiepileptics, other medications such as sulfonamides and allopurinol have been implicated. We report an 18-year-old woman who presented with drug-induced hypersensitivity syndrome secondary to phenytoin.

\section{Case Presentation}

An 18-year-old woman with a newly diagnosed seizure disorder presented to the emergency room with nausea, vomiting, and an erythematous eruption on the face, neck, palms, and soles (Figures 1-4). Three weeks prior to her presentation, she was started on phenytoin $100 \mathrm{mg}$ orally daily. Ten days later, she began to experience pruritis without rash. She visited an urgent care clinic and was prescribed a 5-day prednisone taper. This relieved the itching somewhat. However, she woke up two days later and noticed an erythematous eruption on her face, palms, and soles. She had associated nausea, profuse vomiting, and abdominal pain. The abdominal pain was diffuse, 8 out of 10 in severity, nonradiating, and slightly relieved with analgesics. She denied dysuria or hematuria. She denied sexual activity, recent travel, and recent new foods.

Cutaneous examination revealed erythematous papules coalescing into plaques on the perioral region involving the nasolabial folds (Figure 1). There was a similar distribution on the nape of her neck (Figure 2). She also presented with lesions on her palms and soles that were more focal (Figure 3-4). Her oral mucosa consisted of erosions without vesicles. She was found to have a temperature of $103.1^{\circ} \mathrm{F}$, respiratory rate of 20 , and mild leukocytosis of 11.3. Differential revealed $8.9 \times 10^{3}$ granulocytes/microliter; $1.2 \times 10^{3}$ lymphocytes/microliter, $0.9 \times 10^{3}$ monocytes/microliter, and $0.3 \mathrm{x}$ $10^{3}$ eosinophils/microliter. Her complete metabolic panel was negative. Chlamydia and gonorrhea DNA probes were negative. Monospot was negative. Throat culture revealed usual oral flora. Rapid plasma reagin (RPR) test was negative.

The patient was admitted with a working diagnosis of phenytoininduced exanthem versus enterovirus infection with coxsackie A16 ("hand-foot-and-mouth syndrome"). Stevens-Johnson syndrome was also being considered. A punch biopsy of a right upper arm lesion was performed. The results revealed superficial perivascular dermatitis (Figures 5-6) with focal interface inflammatory component (Figure 7), favoring drug eruption over viral exanthem.

The patient was started on methylprednisolone $40 \mathrm{mg}$ intravenously (IV) every eight hours and diphenhydramine $25 \mathrm{mg}$ IV as needed for itching. She did not enter anaphylaxis and did not require epinephrine or albuterol. Her eruption regressed significantly over three days in the hospital. She was discharged with hydrocortisone $1 \%$ cream to be applied twice daily as well as oral diphenhydramine for pruritis. Her eruption completely cleared two weeks after discharge.

\section{Discussion}


Figure 1. An 18-year-old female presents with erythematous plaques in the perioral region involving the nasolabial folds shortly after starting phenytoin.

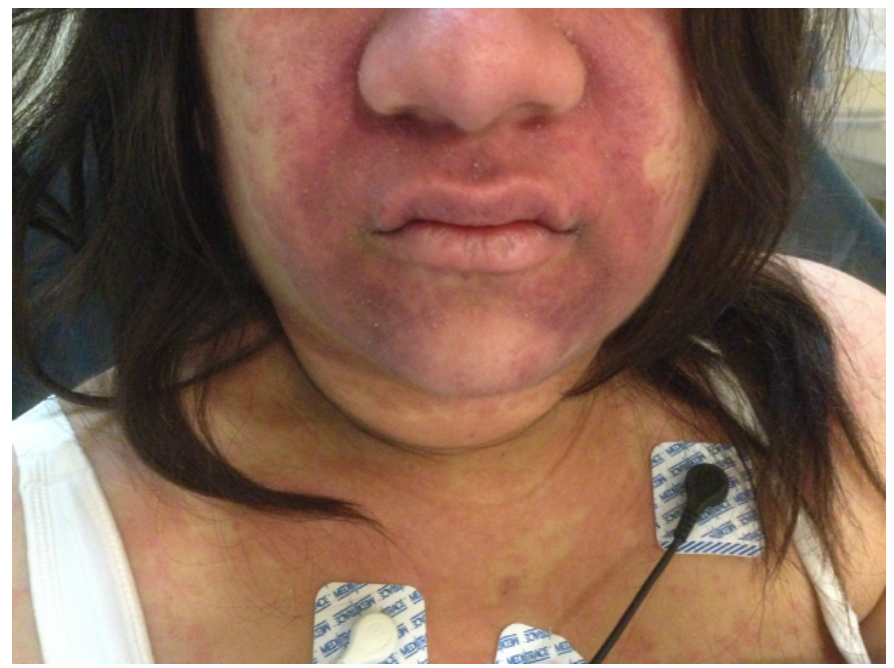

Figure 3. An 18-year-old woman developed erythematous papules on her palms shortly after starting phenytoin.

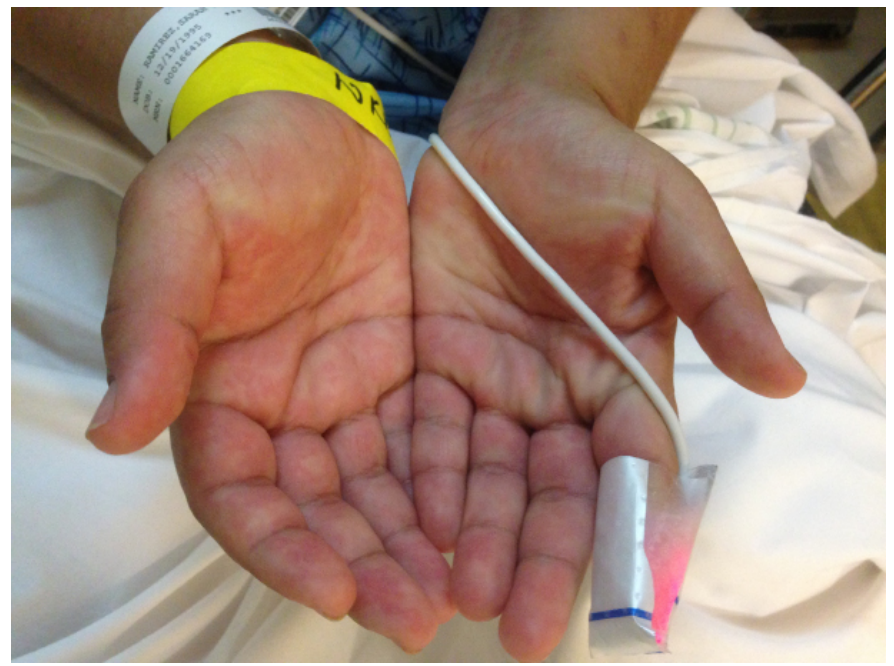

Figure 5. This low-powered hematoxylin and eosin (H\&E) stain demonstrates superficial perivascular dermatitis.

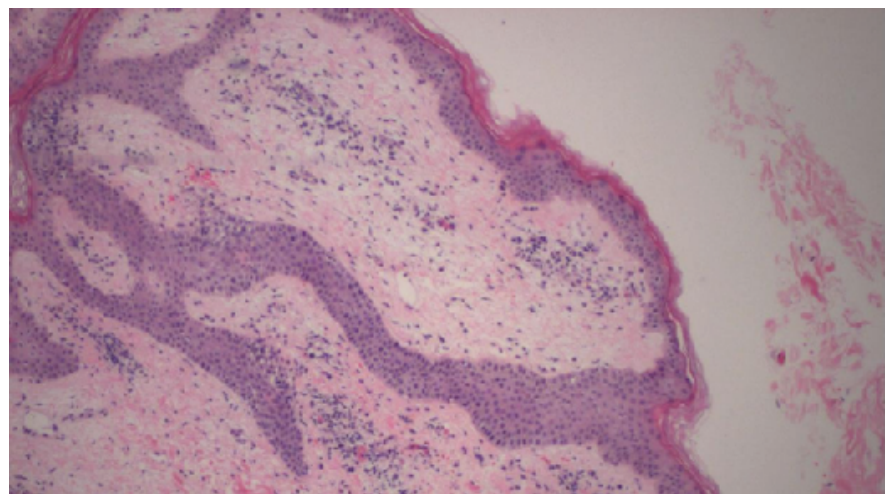

Figure 2. An 18-year-old woman developed erythematous papules coalescing into plaques shortly after starting phenytoin. The lesions on the back of her neck can be seen in this image.

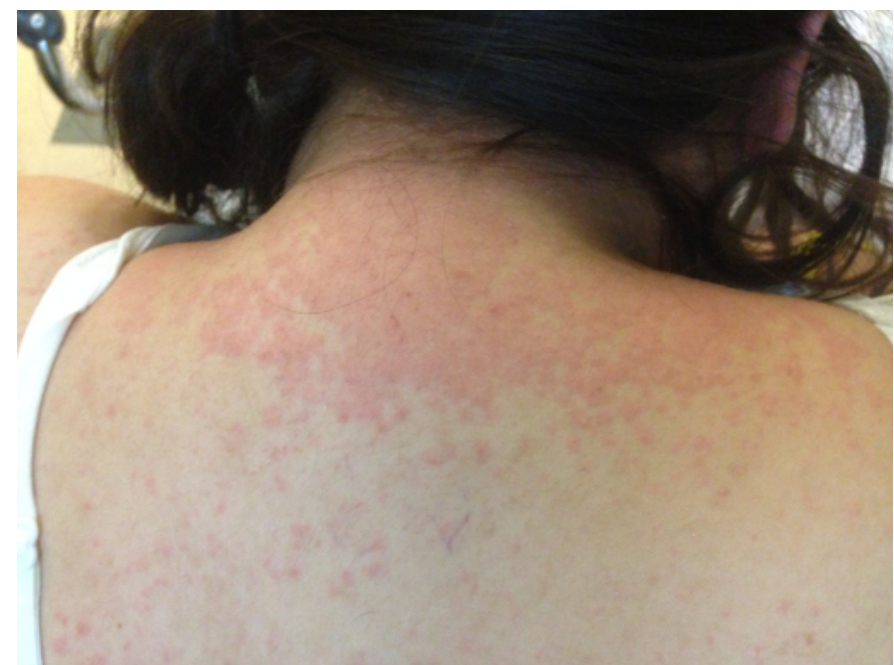

Figure 4. The 18-year-old woman also presents with erythematous papules on her soles.

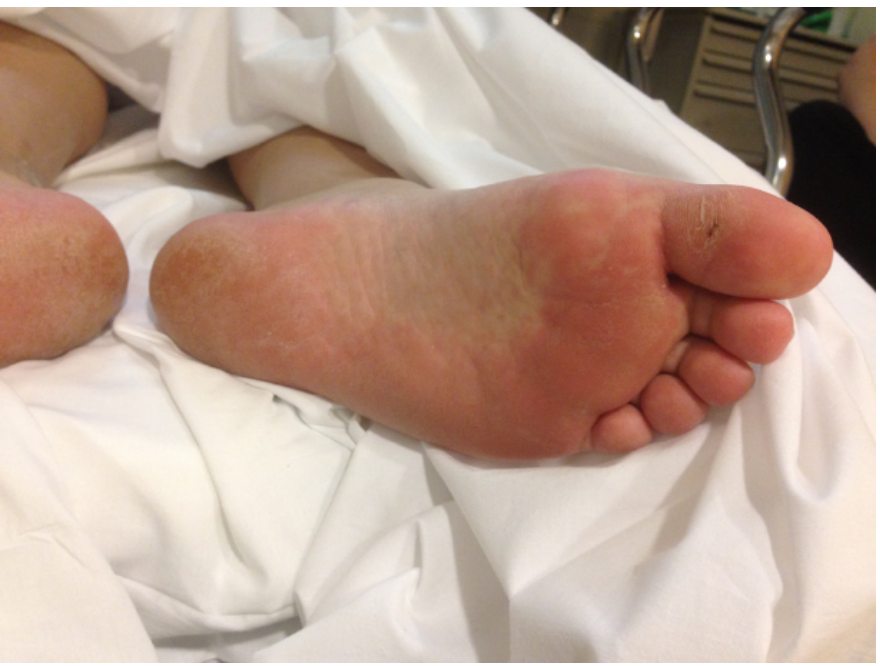

Figure 6. This high-powered H\&E stain further exhibits the superficial perivascular dermatitis.

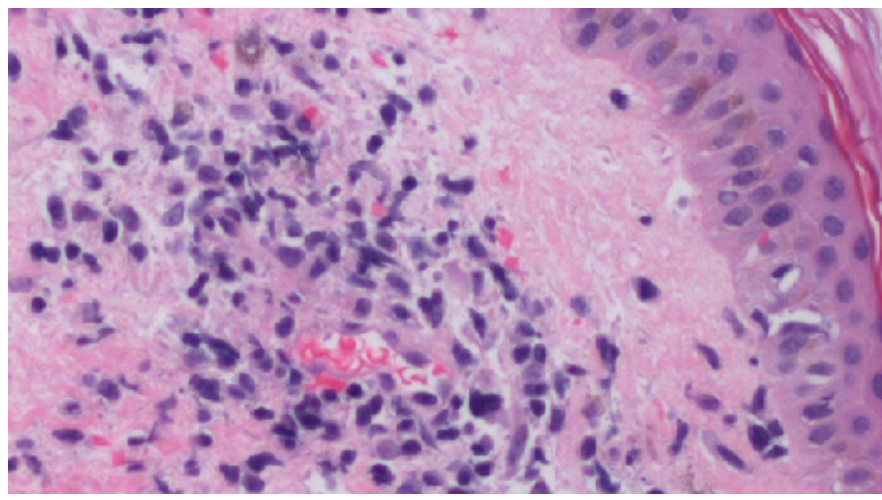


Figure 7. This high-powered H\&E stain shows an interface inflammatory component, inflammation at the dermal-epidermal junction consistent with drug-induced eruption.

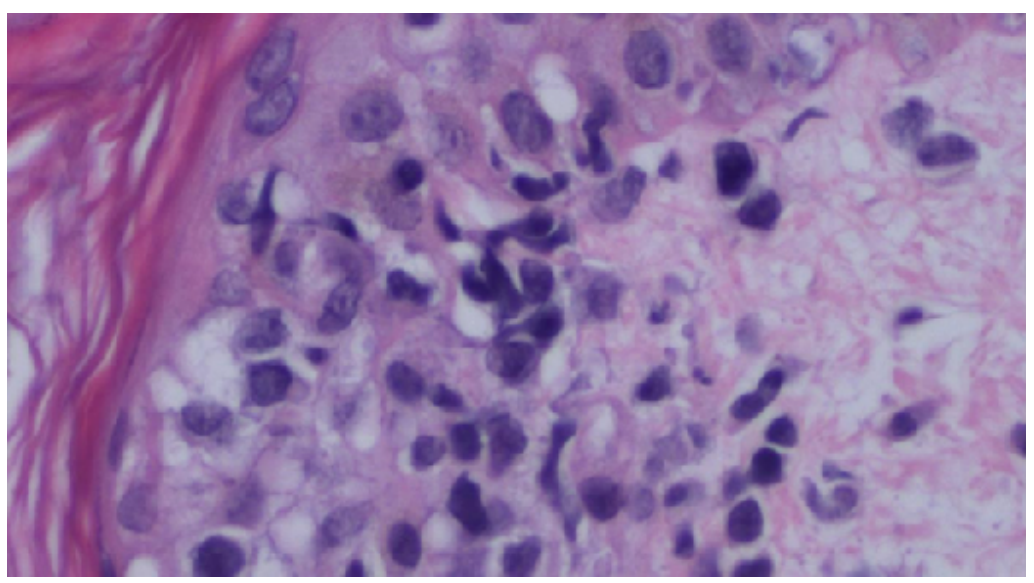

DIHS has been implicated in many drugs. The most common culprits are anticonvulsants (phenytoin, phenobarbital, carbamazepine, oxcarbazepine, and lamotrigine), $64 \%$ of which were estimated in one study [2]. Other causes included allopurinol, dapsone, vancomycin, leflunomide, and nitrofurantoin. Multiple other drugs have been described in the literature. One 14-monthold child developed DIHS due to chloral hydrate [3].

The incidence of DIHS is unknown due to its unpredictable clinical presentation. The onset and timing of DIHS is extremely variable, which has made its report in the literature difficult. Some individuals develop signs and symptoms as early as two weeks, while others may not experience them until 3 months after starting a new antiepileptic medication [1]. Nevertheless, this syndrome has a much longer latency than morbilliform drug eruptions (mean of 10 days) and type- 1 hypersensitivity syndromes (within a day). The liver is the most common organ involved in DIHS, seen in $80 \%$ of patients. This is followed by the kidneys, lungs, heart, muscles, and pancreas [4]. Liver involvement in the form of hepatitis is usually mild but can be severe. The mortality rate is between 18 and $40 \%$ if hepatitis is present [1]. Liver function tests (LFTs) may be extremely elevated and continue to rise even after the drug has been discontinued. It may take up to one year for them to return to baseline. Half of all patients with DIHS have hematological abnormalities. The most common is atypical lymphocytes $(63 \%)$, followed by eosinophilia (52\%), lymphocytopenia $(25 \%)$, thrombocytopenia (25\%), and lymphocytosis (25\%) [4].

The cutaneous findings of DIHS usually begin similarly to a morbilliform drug eruption: erythematous, diffuse macules and papules. These start on the face and trunk. Erythematous facial edema occurs in half of patients. Mucosal involvement of the mouth and pharynx may occur; erosions are rarely seen. $50 \%$ of the body surface area is usually involved [5].

One study of the pharmacogenetics of antiepileptic-induced DIHS investigated associations between HLA alleles and several antiepileptic drugs in diverse populations. The study showed that HLA-B*15:02 was associated with carbamazepine, lamotrigine and phenytoin-induced Stevens-Johnson syndrome in Asian populations. The authors suggest to pretreat such individuals prior to the start of antiepileptic medications [6]. In addition, the pathogenesis of DIHS has been closely linked with herpesvirus reactivation. In a study of 100 patients with DIHS, an increase in the antibody titer against HHV-6 was detected in $60 \%$ of patients two to four weeks after symptom onset [7]. Another study found that EBV, HHV-6, and HHV-7 reactivation ocurred in 29 of 40 patients with DIHS; CD8+ lymphocytes specific to these viruses were found in the blood, skin, and liver [8]. This suggests that DIHS may be due to viral reactivation and cross-reaction of Tcells with the offending drug.

Under light microscopy, DIHS usually reveals nonspecific spongiosis, a perivascular lymphocytic infiltrate with or without eosinophils (Figures $5-6$ ), interface inflammation (Figure 7), and dermal edema [9]. Due to the presence of atypical lymphocytes in the infiltrate, the differential may include cutaneous lymphoma. Biopsy of involved lymph nodes may also demonstrate atypical lymphocytes, but the features are usually benign. In the case of liver involvement, biopsy reveals lobular inflammation, portal inflammation, scattered foci of necrotic hepatocytes, and granulomatous infiltrates containing eosinophils. Portal inflammation and cholestasis also may be seen. Confluent hepatocyte necrosis and lobular disarray due to inflammation and regenerative changes are seen in severe cases. Kidney biopsy reveals tubulointerstitial nephritis (TIN) patterns.

DIHS is a clinical diagnosis. Many criteria have been proposed for diagnosis. RegiSCAR criteria include at least 3 of the following 7 characteristics: 1) skin eruption, 2) fever $\left(>38^{\circ} \mathrm{C}\right)$, 3) lymphadenopathy of at least 2 sites, 4) involvement of at least 1 internal organ, 5) lymphocytosis $\left(>4 \times 10^{3} / \mu \mathrm{L}\right)$ or lymphocytopenia $\left.\left(<1.5 \times 10^{3} / \mu \mathrm{L}\right), 6\right)$ blood eosinophilia $(>10 \%$ or $700 / \mu \mathrm{L})$, and 7$)$ thrombocytopenia $\left(<120 \times 10^{3} / \mu \mathrm{L}\right)$. The 18 -year-old woman presented with fever, lymphopenia, and the exanthem, thus satisfying DIHS by RegiSCAR criteria.

Bocquet's criteria require meeting the following 3 features: 1) skin eruption, 2) blood eosinophilia $\left(>1.5 \times 10^{3} / \mu \mathrm{L}\right)$ or the presence of atypical lymphocytes, and 3) internal organ involvement, including lymphadenopathy $(>2 \mathrm{~cm}$ in diameter), hepatitis (liver transaminases values $>$ twice the upper normal limit), interstitial nephritis, and interstitial pneumonia or carditis.

The Japanese consensus group diagnostic criteria for DIHS includes the following features: 1) eruption of macules and papules developing at least 3 weeks after starting the medication, 2) prolonged clinical symptoms 2 weeks after discontinuing the drug, 3) fever $\left(>38^{\circ} \mathrm{C}\right)$, 4) elevation of liver enzyme (alanine aminotransferase $[\mathrm{ALT}]>100 \mathrm{U} / \mathrm{L}$ ) or involvement of other organs, 5) leukocytosis $\left(>11 \times 10^{3} / \mu \mathrm{L}\right)$, atypical lymphocytosis $(>5 \%)$ or eosinophilia $\left(>1.5 \times 10^{3} / \mu \mathrm{L}\right)$, 6) lymphadenopathy, and 7) human 
herpesvirus (HHV)-6 reactivation. Diagnosis of typical DIHS requires the presence of all 7 criteria. Atypical DIHS is diagnosed in patients with the first five listed criteria.

One study compared patients according to the three criteria. A total of $60.4 \%$ of patients satisfied RegiSCAR definite criteria and $77.1 \%$ satisfied Bocquet's criteria. Only $18.8 \%$ satisfied atypical DIHS criteria from the Japanese group. They concluded that Bocquet's criteria is most appropriate because of its efficiency in clinical practice. Lymphocyte and eosinophil counts, as well as creatinine and ferritin levels, could be useful early prognostic factors [10].

The differential diagnosis of DIHS includes cutaneous T-cell lymphoma, morbilliform drug eruption, and Stevens-Johnson syndrome / toxic epidermal necrolysis (SJS/TEN). Unlike DIHS, cutaneous T-cell lymphoma presents with generalized erythroderma. Unlike DIHS, SJS presents more commonly with oral mucosal involvement and rarely presents with eosinophilia or atypical lymphocytes. Histology of SJS shows full-thickness epidermal necrosis.

The primary management of DIHS is to remove the offending drug. If the manifestation is not severe enough to result in end organ involvement, then symptomatic treatment is preferred: antihistamines for pruritis and topical corticosteroids for skin manifestations. The most effective treatment for DIHS with organ involvement is systemic corticosteroids. In one study of 17 patients, 13 patients treated with oral prednisone recovered completely, two developed post-inflammatory hyperpigmentation (PIH), one developed renal failure, and one patient developed liver failure [2]. It is important to note that systemic corticosteroids have had no proven benefit for liver manifestations of DIHS. Nor has a treatment duration been definitively determined.

Post-treatment monitoring has not been clearly outlined in the literature, but it is suggested to perform weekly blood work to assess for end organ involvement and resolution: complete blood count with differential and complete metabolic panel. The prognosis of this disease is usually favorable, with only a five to $10 \%$ mortality that is often related to severe end organ involvement [11].

\section{Conclusion}

Anticonvulsant drug-induced hypersensitivity syndrome (DIHS) is a rare but sometimes fatal disorder. It is characterized by fever, rash, hepatitis, and other multiorgan involvement. Implicated anticonvulsants include phenytoin, carbamazepine, phenobarbital, and lamotrigine. The incidence of DIHS is unknown due to its unpredictable clinical presentation. Onset occurs between 2 weeks and 3 months of starting the offending agent. Studies of genetics and heritability propose an association with HLAB*15:02. Three diagnostic criteria include RegiSCAR, Bocquet's, and the Japanese consensus group diagnostic criteria for DIHS. Removal of the offending agent and symptomatic treatment are the mainstay of management. An 18-year-old woman presented with nausea, vomiting, and an erythematous eruption on the face, neck, palms, and soles two weeks after starting phenytoin. Her symptoms resolved after a short hospitalization, systemic corticosteroids, and subsequent symptomatic topical treatment.

\section{References}

[1]. Havill S, Rademaker M. Anticonvulsant hypersensitivity syndrome. Prescriber Update 16: 28-31. http://www.medsafe.govt.nz/profs/puarticles/6. htm (accessed 10 June 2014)

[2]. Sultan SJ, Sameem F, Ashraf M. Drug reaction with eosinophilia and systemic symptoms: manifestations, treatment, and outcome in 17 patients. Int J Dermatol. 2014 Apr 16. doi: 10.1111/ijd.12331. [Epub ahead of print] PubMed PMID: 24738653.

[3]. Yoo SD, Kim SG, Kim SH, Kim HY. Drug rash with eosinophilia and systemic symptoms syndrome induced by chloral hydrate in early childhood. Allergy Asthma Immunol Res. 2014 May;6(3):270-2. doi: 10.4168/ aair.2014.6.3.270. Epub 2013 Dec 10. PubMed PMID: 24843805; PubMed Central PMCID: PMC4021248.

[4]. Walsh SA, Creamer D (January 2011). Drug reaction with eosinophilia and systemic symptoms (DRESS): a clinical update and review of current thinking. Clinical and Experimental Dermatology 36 (1): 6-11. doi:10.1111/ j.1365-2230.2010.03967.x.PMID 21143513

[5]. Kardaun SH, Sekula P, Valeyrie-Allanore L, Liss Y, Chu CY, Creamer D, Sidoroff A, Naldi L, Mockenhaupt M, Roujeau JC, RegiSCAR study group. Drug reaction with eosinophilia and systemic symptoms (DRESS): an original multisystem adverse drug reaction. Results from the prospective RegiSCAR study. Br J Dermatol. 2013;169(5):1071.

[6]. Bloch KM, Sills GJ, Pirmohamed M, Alfirevic A. Pharmacogenetics of antiepileptic drug-induced hypersensitivity. Pharmacogenomics. 2014 Apr;15(6):857-68. doi: 10.2217/pgs.14.65. PubMed PMID: 24897291.

[7]. Tohyama M, Hashimoto K, Yasukawa M, Kimura H, Horikawa T, Nakajima K, Urano Y, Matsumoto K, Iijima M, Shear NH, Association of human herpesvirus 6 reactivation with the flaring and severity of drug-induced hypersensitivity syndrome. Br J Dermatol. 2007 Nov;157(5):934-40. Epub 2007 Sep 13.

[8]. Picard D, Janela B, Descamps V, D'Incan M, Courville P, Jacquot S, Rogez S, Mardivirin L, Moins-Teisserenc H, Toubert A, Benichou J, Joly P, Musette P. Drug reaction with eosinophilia and systemic symptoms (DRESS): a multiorgan antiviral T cell response. Sci Transl Med. 2010;2(46):46ra62.

[9]. Chi MH, Hui RC, Yang CH, Lin JY, Lin YT, Ho HC, Chung WH, Kuo TT. Histopathological analysis and clinical correlation of drug reaction with eosinophilia and systemic symptoms (DRESS). Br J Dermatol. 2014 Apr;170(4):866-73.

[10]. Kim DH, Koh YI. Comparison of diagnostic criteria and determination of prognostic factors for drug reaction with eosinophilia and systemic symptoms syndrome. Allergy Asthma Immunol Res. 2014 May;6(3):216-21. doi: 10.4168/aair.2014.6.3.216. Epub 2014 Feb 6.

[11]. Chen YC, Chiu HC, Chu CY. Drug reaction with eosinophilia and systemic symptoms: a retrospective study of 60 cases.Arch Dermatol. 2010 Dec;146(12):1373-9. Epub 2010 Aug 16. 